\title{
DIE ALMA-GEMEINSCHAFT IN CORONA-ZEITEN
}

\author{
von Susanne Luger
}

Zusammenfassung: Die Pandemie und die damit verbundenen Maßnahmen und Einschränkungen trafen alle Kolleg*innen der Alma-Gemeinschaft gleichermaßen und brachte Veränderungen mit sich. Manche Bereiche einer Bibliothek traf es dabei mehr als andere. Während Kolleg*innen, die im Bereich der E-Medien-Verwaltung tätig sind, leichter von zu Hause aus arbeiten konnten, oft mit noch weiterreichenden Arbeitsaufträgen ausgestattet als im Büro, wurde Leih- und Lesesaalbetrieb am Beginn der Pandemie gänzlich heruntergefahren, mit weniger Möglichkeit in der Homeoffice-Situation. Die seit Beginn des Alma-Umstieges regelmäßig stattfindenden Webcalls der Alma-Fachexpert*innengruppen boten den Kolleg*innen in der durch den Lockdown oft sehr isolierten Welt mehr denn je eine Plattform der Kommunikation, des Austausches, als auch des Rückhaltes aufberuflicher ebenso wie persönlicher Ebene. Wie erging es also den Kolleg*innen? Über welche Themen wurde gesprochen? Wie konnten sie sich gegenseitig unterstützen? Ein Bericht aus dem Alma-Forum.

Schlagwörter: Alma; Bibliothekar*in; Lockdown; Webcall; Kommunikation

\section{THE ALMA-COMMUNITY IN TIMES OF CORONA}

Abstract: The pandemic and the necessary measures that had to be taken have had an impact on all colleagues in the Austrian Alma community alike, some more than others. While some colleagues, like those in e-resource management, had it easier working from home, often with more workload than before, those working in fulfillment had fewer possibilities due to the closing of circulation desks and reading rooms. Since the start of the implementation of Alma there have been regular webcalls between the functional experts, which in times of isolation during the lockdown have offered them more than ever a platform for communication, exchange and support not only professionally but also on a personal level. How were the colleagues faring? What topics were discussed? In which way could they help each other? A report from the Alma-Forum.

Keywords: Alma; librarian; lockdown; web call; communication

DOI: https://doi.org/10.31263/voebm.v73i3-4.5333

(c) Susanne Luger

Dieses Werk ist - exkl. einzelner Logos und Abbildungen - lizenziert unter einer Creative-Commons-Lizenz Namensnennung 4.0 International-Lizenz 
Die Pandemie, der damit einhergehende Lockdown und die Schließungen der Bibliotheken stellte viele Kolleg*innen vor gravierende Änderungen im beruflichen, aber auch privaten Leben. Die Arbeit wurde vielfach ins eigene Heim verlegt, Kinder zuhause unterrichtet und die Möglichkeit, den eigenen vier Wänden zu entkommen, eingeschränkt. Eine Neuerung aber war für so manche schon ein alter Hut: Webcalls.

Von Beginn an, als die ersten Bibliotheken in Österreich in die Implementierungsphase von Alma starteten, trafen sich Kolleg*innen der verschiedenen Arbeitsbereiche virtuell über Webcalls, um sich über Alma auszutauschen. Diese Gemeinschaft wuchs mit der Anzahl an Bibliotheken, die über die letzten Jahre ihr Bibliothekssystem wechselten. Während die Schwierigkeiten mit Alma aufgrund der fortlaufenden kleinen und größeren Änderungen im System und den sich damit ergebenden Fragestellungen weiterhin Gesprächsstoff lieferten, gab es nun ein zusätzliches Thema, worüber man sich austauschen konnte und wollte.

Die Maßnahmen, die aufgrund steigender Infektionszahlen gesetzt wurden, führten im ersten Moment zu Verunsicherung und Skepsis, doch hinderte es die Kolleg*innen nicht daran, sich gegenseitig zu motivieren und sie bemühten sich sehr, den Betrieb mit den zur Verfügung stehenden Mitteln aufrecht zu erhalten und den Nutzer*innen weiterhin den in dieser Situation bestmöglichen Service zu bieten. Die Herausforderungen und der Austausch über die Webcalls fanden in jedem Bereich in unterschiedlichem Ausmaß statt, weshalb hier nachfolgend kurze Einblicke in die jeweiligen Gruppen gegeben werden soll.

ACQ: Die Acquisitions-Gruppe (Erwerbung) hatte coronabedingt an mehreren Fronten zu kämpfen. Lieferanten, die nicht liefern konnten, gehörten dabei ebenso dazu wie stark rückläufige Bestellwünsche für Printmedien und die damit einhergehende Verlagerung auf die Erwerbung elektronischer Medien. Es gab erste Versuche in EBS (Evidence Based Subscription) und eine „Digitalisierung“ der Arbeitsabläufe, z. B. fand die Rechnungsbearbeitung im Homeoffice Einzug. Demnach mussten die Kolleg*innen rasch und flexibel auf die geänderten Umstände reagieren, was auch in den Webcalls kurz besprochen wurde.

ER: Die Expert*innengruppe E-Medien hielt während der Lockdownphase sowohl über Webcalls als auch über die Mailingliste Kontakt, um sich auch noch zusätzlich sowohl inhaltlich wie auch verwaltungstechnisch über die durch die COVID-19-Phase ausgelösten frei verfügbaren Verlagsangebote auszutauschen. Es wurden nicht nur freie elektronische Ressourcen rund um das Thema COVID-19, sondern auch z. B. für eine eingeschränkte Zeitspanne frei verfügbare elektronische Bücher von vie- 
len Verlagen angeboten. Jede Bibliothek war darum bemüht, unter den erschwerten Bedingungen die bestmögliche Literaturversorgung für alle Bibliotheksnutzer*innen zu gewährleisten, was in vielen Fällen eine Erweiterung des uneingeschränkten elektronischen Ressourcenangebots nicht nur für die Forschung, sondern vor allem auch für die digitale Lehre zur Folge hatte. Damit ging unter anderem auch ein erhöhter Bedarf an Auskünften bis hin zu Schulungen zum Thema elektronische Ressourcen einher, was von etlichen Kolleg*innen aus der Expert*innengruppe E-Medien übernommen wurde.

FUL: Die Fulfillment-Gruppe (Entlehnung und Benutzung) war seit März wohl jene mit dem größten Zulauf an Teilnehmer*innen und dem regsten Austausch. Corona und die damit verbundenen Maßnahmen in den jeweiligen Häusern dominierte die Calls der Kolleg*innen. Die Frequenz der ursprünglich monatlich stattfindenden Termine, in denen zumeist nur kurz die Release Notes besprochen wurden, wurde bald erhöht, um schneller über die letzten Entwicklungen diskutieren zu können. Die Protokolle der vergangenen Calls zeigen, welche Ideen die Kolleg*innen hatten und welch kreative Lösungen sie den Nutzer*innen anboten, um diese weiterhin trotz geschlossener Lesesäle und Leihstellen mit Literatur versorgen zu können. Auch zu den möglichen Arbeitsaufträgen im Homeoffice oder den unterschiedlichen Vorgehensweisen bei der schrittweisen Öffnung der Bibliotheken fand ein intensiver Austausch statt.

RM: Die Resource-Management-Gruppe (Formal- und SacherschlieBung) erfuhr wie schon die Fulfillment-Gruppe im ersten Lockdown einen Zuwachs bei ihren Webcalls, die seit Anbeginn zweiwöchentlich stattfinden. Wie für viele andere Arbeitsbereiche in einer Bibliothek war im Resource-Management die Arbeit im Homeoffice eine große Herausforderung, die bereits bei der Technik begann. Die erste Verunsicherung wich schon bald Vorschlägen und Ideen, welche Aufgaben gut von zu Hause aus erledigt werden könnten und man tauschte sich über die neuen Arbeitsabläufe in den einzelnen Häusern aus. Die ersten paar Minuten jedes Calls wurden seither immer einem kurzen Bericht aus den Bibliotheken gewidmet, bevor in medias res gegangen wurde.

RS: Die Resource-Sharing-Gruppe (Fernleihe), die aufgrund ihrer Arbeit von jeher vernetzter war als manch andere, kommunizierte bisher nur anlassbezogen über Webcalls und nutzte stattdessen sehr stark den Austausch über die E-Mail-Liste, da hierüber auch Nicht-Alma-Bibliotheken erreicht, benachrichtigt und um Rat gefragt werden können. Denn auch an der Fernleihe ging Corona nicht spurlos vorüber. Die zeitweise Einstellung des Leih- und Lesesaalbetriebes führte folglich zu Anpassungen im 
Fernleihverkehr und Leihfristen, welche zeitnah an die Kolleg*innen weitergegeben werden mussten. Und obwohl die Kommunikation über E-Mail gut funktioniert, finden seit Herbst wieder regelmäßige Calls statt, da der persönliche Kontakt doch eine willkommene Abwechslung darstellt.

ZS: Corona war natürlich auch in der Zeitschriften-Gruppe ein neuer Tagesordnungspunkt neben den üblichen Alma-Zeitschriften-Themen. Ohne Zugang zu den Zeitschriften im Homeoffice blieb den Bearbeiter*innen meist nichts anderes übrig, als Datenbereinigungen vorzunehmen, wodurch die Webcalls eine angenehme Abwechslung darstellten, auch um zu erfahren, wie es den Kolleg*innen in dieser Zeit erging und wie die Situation in den anderen Bibliotheken aussieht. Wahrscheinlich konnte deshalb ein gestiegenes Interesse und ein Zuwachs an Teilnehmer*innen bei den virtuellen Treffen festgestellt werden.

Covid-19, dem Lockdown und seinen Folgen lässt sich somit durchaus etwas Positives abgewinnen. Die Alma-Gemeinschaft ist nicht nur zahlenmäßig gewachsen, sondern auch näher zusammengerückt. Die Webcalls der Fachexpert*innengruppen halfen den Kolleg*innen einerseits sich in der neuen Situation zurechtzufinden und zu unterstützen und andererseits sich gegenseitig aufzumuntern. Sie boten ihnen in der sonst sehr isolierten Welt der Datenbereinigung Abwechslung und mehr denn je eine Plattform der Kommunikation und des Austausches.

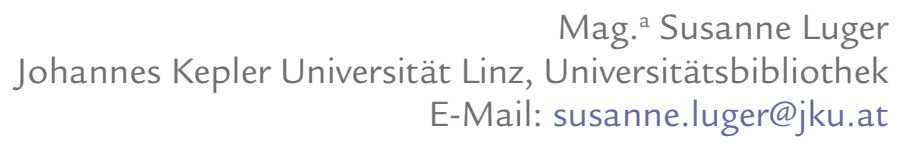

